\title{
Special feature: functional data analysis and its applications
}

\author{
Gil González-Rodríguez ${ }^{1} \cdot$ Hidetoshi Matsui $^{2}$
}

Published online: 21 March 2019

(c) The Behaviormetric Society 2019

Recent advances in high-throughput measurement technologies and devices make the availability of large-scale data sets requiring new statistical methodologies for their analysis. In particular, more and more time-course data have been obtained which can be modeled as functions.

Functional data analysis has received considerable attention in different fields of application, including bioscience, medical science, and meteorology. One of the ideas behind functional data analysis is to express time-course data as smooth functions and then draw information from the collection of functional data. Functions are observed on a finite grid, and one important advantage is that data can be analyzed even when the number of grid points and its concrete values vary from individual to individual. This special issue focuses on functional data analysis and its applications in various fields where time-course data can be available. The aim is to illustrate the effectiveness of the methodologies to analyze functional data through their applications.

This issue consists of one invited and three contributed papers. The invited paper by Araki and Kawaguchi (2019) "Functional logistic discrimination with sparse PCA and its application to the structural MRI" proposes a supervised classification method to handle high-dimensional image predictors by combining sparse principal components. The three contributed papers are as follows: Misumi et al. (2019) paper "Multivariate functional clustering and its application to typhoon data" proposes a multivariate nonlinear mixed effects model for clustering multiple longitudinal data and applies the method to the analysis of typhoon data. Takagishi and Yadohisa (2019) paper "Robust curve registration using the $t$ distribution" proposes a new registration method for a functional data set and then applies it to the electrocardiogram data analysis. Bouanani et al. (2019) paper "Asymptotic normality of

Hidetoshi Matsui

hmatsui@biwako.shiga-u.ac.jp

Gil González-Rodríguez

gil@uniovi.es

1 Department of Statistics and INDUROT, University of Oviedo, Oviedo, Spain

2 Faculty of Data Science, Shiga University, Shiga, Japan 
some conditional nonparametric functional parameters in high-dimensional statistics" considers the theoretical properties of the functional regression model and then applies the method to the analysis of data on spectrometric study.

\section{References}

Araki Y, Kawaguchi A (2019) Functional logistic discrimination with sparse PCA and its application to the structural MRI. Behaviormetrika. https://doi.org/10.1007/s41237-019-00079-3

Bouanani O, Laksaci A, Rachdi M, Rahmani S (2019) Asymptotic normality of some conditional nonparametric functional parameters in high dimensional statistics. Behaviormetrika. https://doi. org/10.1007/s41237-018-0057-9

Misumi T, Matsui H, Konishi S (2019) Multivariate functional clustering and its application to typhoon data. Behaviormetrika. https://doi.org/10.1007/s41237-018-0066-8

Takagishi M, Yadohisa H (2019) Robust curve registration using the $t$ distribution. Behaviormetrika. https://doi.org/10.1007/s41237-019-00077-5 